\section{Reply to Professor Yerushalmy's letter on Congenital Heart Disease}

WE were greatly interested in Professor Yerushalmy's data, for it is only by collecting information from many different sources that a finding can be substantiated epidemiologically.

Obviously any study in which, for example, the smoking history was obtained during pregnancy rather than after delivery is at an advantage. We believe, however, that the type of bias inherent in any retrospective survey is unlikely to have occurred to any marked extent in the present series because at the time of the inquiry there had been no publicity concerning the effects of smoking in pregnancy.

It is difficult to find any explanation (other than that due to chance) of the differences between the incidences of congenital heart disease (CHD) in the two populations of births. We would like to point out, though, that in our paper we had a second series of 204 cases of CHD among 7,000 perinatal deaths, and that the differences in incidence of CHD between infants of smokers and non-smokers were of the same order of magnitude as in our population series, even when variables such as maternal age, parity and social class had been allowed for.

As to the effect of smoking on perinatal mortality in general, we are somewhat surprised at Yerushalmy's interpretation of his Table 2 in which he states that the perinatal mortality rate in general is not larger for infants of mothers who smoke. In fact, of the selected studies he reports, and excluding Yerushalmy's 1964 sample which is a subsample of the 1971 sample, three studies show a very small increase in perinatal mortality and two studies show a large increase. Furthermore, he fails to mention the fact that all these studies give a similar estimate $(150-200 \mathrm{~g})$ for the average reduction in birthweight associated with smoking in pregnancy. Other studies ${ }^{1}$ have shown that the reduction in gestation is only marginal, and we conclude that the effect of smoking is to produce a degree of foetal growth retardation. It is, however, irrelevant to compare the mortality rates of all infants under $2,500 \mathrm{~g}$. Growth retardation although carrying a high risk of perinatal death is entirely different from low birth weight due to immaturity. The latter carries an even higher perinatal mortality death rate, and must obviously be considered separately.

\section{J. FEDRICK}

Regius Professor of Medicine, University of Oxford

Paediatric Research Unit, Guy's Hospital, London

E. D. Alberman

\section{National Child Development Study,}

\section{London}

1 Butler, N. R., Goldstein, H., and Ross, E. M., Brit. Med. J., 2, 127 (1972).

\section{Marginal Vitamin $\mathbf{B}_{12}$ Intake during Gestation in the Rat has Long Term Effects on the Offspring}

IT is now evident that malnutrition of the foetus or of the infant results in long term abnormalities in the physical, clinical and behavioural characteristics of the organism ${ }^{1-4}$. Experimental studies have generally dealt with the effects of severe malnutrition. There is reason to believe that less severe dietary imbalances, more likely to occur in population groups of the Western world, if imposed at an early stage, may have profound, adverse effects much later in life. Because the nutrient demands per unit of body weight are presumably higher in the foetus than in the newborn, a diet which is considered fully adequate for post-natal development does not necessarily provide, when consumed by the mother, an adequate nutritional environment for the developing foetus.

In previous studies ${ }^{5}$ we observed that a high dietary level of $B_{12}$ resulted in increased weight of the newborn compared with that of offspring from mothers receiving a basal diet in which the $\mathbf{B}_{12}$ level was somewhat in excess of the estimated needs for pregnant rats ${ }^{6}$. This latter dietary $B_{12}$ level supports a normal or adequate birth weight in rats and permits a rate of post-natal growth which is considered to be within acceptable limits. However, based on the birth weight differences, our results suggested that the level of $\mathbf{B}_{12}$ in the basal diet represented a marginal deficiency of the vitamin and so we have explored its long term effects on the wellbeing of the offspring.

Pregnant rats received a dietary $B_{12}$ intake $(50 \mu \mathrm{g} / \mathrm{kg}$ diet $)$ which somewhat exceeded the estimated requirement for the pregnant, lactating and growing rat $^{6}$, but which approximated a concentration often used in purified diets, or a level in considerable excess of this intake $(50,000 \mu \mathrm{g} / \mathrm{kg}$ diet $)$. Female rats (Sprague-Dawley, Charles River Laboratories) were housed in screen-bottom cages, given water ad lib. and fed from weanling on a basal diet. This diet contained (percentage by weight: $20.0 \mathrm{C}$-1 assay soybean protein; 28.9 glucose; 28.5 sucrose; cotton seed oil 15 ; choline 0.3 ; DL-methionine 0.3 ; salt mix 5.0; vitamin mix 2.0. Further details of diet have been described ${ }^{7}$. A supplement of crystalline $\mathrm{B}_{12}$ was added to the diet, at the time it was mixed, to achieve a concentration of $50 \mu \mathrm{g} / \mathrm{kg}$ of diet. At maturity, the female rats were placed in breeding units, three animals per cage. They continued to receive the basal, (control) diet and were bred to normal male rats of the same strain. Breeding was confirmed by a positive vaginal sperm smear. At this time, the female rats were transferred to individual cages and they were randomly allocated to receive the basal diet or the higher supplemental vitamin $B_{12}$ level $\left(50 \times 10^{3} \mu \mathrm{g} / \mathrm{kg}\right.$ diet $)$. Mean food intake during the 21-day gestation period was essentially the same in both groups: $454 \mathrm{~g}$ and $461 \mathrm{~g}$ for the basal and high $\mathrm{B}_{12}$ groups, respectively. Similarly, mean total weight gain during this period was $135 \mathrm{~g}$ and $128 \mathrm{~g}$ for the basal and high $\mathbf{B}_{12}$ groups, respectively. Two days after birth, litter size was reduced to eight and the mother was then fed the control diet until the young were weaned at 21 days. After weaning, the progeny continued to receive basal diet until they were used for various measurements. Although the higher levels of dietary $\mathrm{B}_{12}$ were given only during gestation it is recognized that the $\mathbf{B}_{\mathbf{1 2}}$ supplied to the offspring born to mothers receiving the higher supplement of $\mathbf{B}_{12}$ most probably remained higher during lactation than those born to mothers given the control diet. Offspring were taken at various periods during the first year of life for assay of liver aminopyrine demethylase and glucose6-phosphatase (EC3139) activity ${ }^{8}$.

The latter was also visualized by histochemical means in both liver and kidney ${ }^{9}$. Enzyme assays were conducted on the livers of year-old rats which were fasted overnight and tissues collected for histological assessment ${ }^{10}$. To assess the possible clinical benefit gained from the increased $\mathbf{B}_{12}$ intake we utilized an experimental model previously described for study of the metabolic effects of a bacterial infection ${ }^{11}$. Rats were infected by oral administration with $5 \times 10^{9}$ organisms of Salmonella typhimurium at 3 months of age. Liver levels of $B_{12}$ were determined ${ }^{13}$ in representative female rats at time of conception and at littering and in the newborn for each diet group.

In agreement with our earlier findings, the higher dietary level of $\mathbf{B}_{12}$ during gestation did not affect litter size but significantly increased birth weight (Table 1). Body weight differences were still evident after $1 \mathrm{yr}$, demonstrating a long term influence of the higher dietary level of $B_{12}$ provided during intrauterine life. Determinations of total body nitrogen and lipid revealed that the weight difference among the two offspring groups was reflected in a greater proportion of total 